\title{
Melatonin Increasingly Used in Hospitalized Patients
}

\author{
Thomas E MacMillan, MD, MSc, FRCPC1,2,3*, Philip Lui, BScPhm, PharmD4, \\ Robert C Wu, MD, MSc, FRCPC $2,3,5$, Rodrigo B Cavalcanti, MD, MSc, FRCPC $1,2,3$
}

${ }^{1}$ Division of General Internal Medicine, Toronto Western Hospital, University Health Network, Toronto, Ontario, Canada; ${ }^{2}$ Division of General Internal Medicine, Department of Medicine, University of Toronto, Toronto, Ontario, Canada; ${ }^{3}$ HoPingKong Centre for Excellence in Education and Practice, University Health Network, Toronto, Ontario, Canada; ${ }^{4}$ Department of Pharmacy, Toronto Western Hospital, University Health Network, Toronto, Ontario, Canada; ${ }^{5}$ Division of General Internal Medicine, Toronto General Hospital, University Health Network, Toronto, Ontario, Canada.

The frequency of melatonin use for insomnia in hospitalized patients is unknown. This study assessed temporal trends of melatonin use in the hospital and compared them with those of use of zopiclone and lorazepam. We performed a retrospective observational study over 6 years from January 2013 to December 2018 at two academic urban hospitals in Toronto, Canada. We abstracted pharmacy dispensing data and standardized rates of medication use by inpatient days. Melatonin use increased from almost none to more than 70 doses per
1,000 inpatient days during 2013-2018, while zopiclone use decreased by 20 doses per 1,000 inpatient days. Melatonin use was twice as high at one hospital and was higher on internal medicine and critical care. Overall use of the three medications increased by $25.7 \%$, which mainly reflects a marked increase in melatonin use. Melatonin is likely being used in a proportion of patients who would not otherwise have received a sleep medication. Journal of Hospital Medicine 2020;15:349-351. (C) 2020 Society of Hospital Medicine leep disturbance is common in hospitals, and both the quality and quantity of sleep are negatively affected in hospitalized patients. ${ }^{1}$ Sleep disturbances in hospitals are associated with hyperglycemia, ${ }^{2}$ delirium, ${ }^{3}$ lower patient satisfaction, ${ }^{4}$ and increased risk of readmission. ${ }^{5}$

A significant proportion of hospitalized patients receive sleep medications (ie, hypnotic medication) despite limited evidence. ${ }^{6,7}$ Sleep medications have adverse effects including falls, fractures, cognitive impairment, and delirium. ${ }^{8}$ Commonly used nonbenzodiazepine sleep medications (eg, zopiclone) are perceived as safer but may have similar risks. ${ }^{9}$

Melatonin is increasingly used to treat insomnia, although evidence for its efficacy and safety is lacking. ${ }^{10}$ While melatonin use doubled between 2007 and 2012 in the United States, ${ }^{11}$ previous hospital-based studies have not included melatonin. ${ }^{7}$ It is not known if increased melatonin use in the hospital mitigates use of higher-risk medications. Melatonin preparations can also have quality issues, including deviations from labelled dosage and contamination with compounds such as serotonin, ${ }^{12}$ and patients continuing melatonin after discharge could have adverse effects if switched to different preparations.

In this study, we aimed to determine temporal trends in mel-

*Corresponding Author: Thomas E MacMillan, MD, MSc, FRCPC; Email: tom.macmillan@uhn.ca; Telephone: 416-603-5800; Twitter: @MacMillanTom. Published online first May 20, 2020

Find additional supporting information in the online version of this article.

Received: December 2, 2019; Revised: March 2, 2020;

Accepted: March 8, 2020

๑ 2020 Society of Hospital Medicine DOI 10.12788/jhm.3408 atonin use in hospitalized patients, and compare them with trends in use of other sleep medications.

\section{METHODS}

We conducted the study at two urban academic hospitals with a total of 706 acute care beds in the same network in Toronto, Canada. This study was approved by the University Health Network's research ethics board.

We abstracted pharmacy dispensing data on melatonin, zopiclone, and lorazepam from January 1, 2013, to December 31, 2018. We included oral medications dispensed to inpatient units or admitted patients in the emergency department (ED). Zopiclone is the most commonly used nonbenzodiazepine sleep medication in Canada. ${ }^{13}$ Lorazepam is the most commonly used benzodiazepine at our institution ( $97 \%$ of all oral benzodiazepine doses). While lorazepam is prescribed for many nonsleep indications, we included it to assess the impact of melatonin. We did not include antipsychotics or trazodone, which are rarely newly initiated for insomnia at our institution.

We abstracted the monthly number of doses dispensed by unit and hospital. We categorized units based on the primary patient population as either internal medicine, critical care, or other. Admitted patients in the ED were counted as "other" regardless of service. As the focus of our study was on internal medicine and critical care, we did not analyze by type of unit in the "other" group, which is heterogeneous.

Each medication-dispensing event was counted as one dose, regardless of the number or strength of tablets (eg, a patient dispensed two 3-mg tablets of melatonin, for a total of $6 \mathrm{mg}$, would be counted as a single dose). Most unused doses are credited back (ie, if a medication was refused and returned to pharmacy, it 
TABLE. Yearly Rate of Doses Dispensed per 1000 Inpatient Days of three Study Medications from January 2013 to December 2018

\begin{tabular}{lccc}
\hline \multicolumn{3}{c}{ per 1000 inpatient days by medication } \\
\hline 2013 & Melatonin & Zopiclone & Lorazepam \\
\hline 2014 & 0.4 & 109.9 & 101.8 \\
\hline 2015 & 6.5 & 107.5 & 98.4 \\
\hline 2016 & 18.8 & 107.2 & 95.7 \\
\hline 2017 & 35.8 & 96.2 & 87.5 \\
\hline 2018 & 43.5 & 89.6 & 99.7 \\
\hline Absolute change & 71.7 & 89.5 & 105.3 \\
\hline (2018 vs 2013) & 71.4 & -20.4 & 3.5 \\
\hline
\end{tabular}

was not counted). Lorazepam and zopiclone are on hospital formulary, while melatonin is not. To order melatonin, clinicians must select "Nonformulary medication" in the electronic health record and manually enter medication name, dose, route, and frequency, as well as select a justification for use. The hospital supplies nonformulary medications such as melatonin to patients.

To account for changes in patient volumes, we standardized medication dispensing rates per 1,000 inpatient days. We discovered rare instances in which the monthly number of doses was a negative number because of pharmacy inventory accounting. This issue affected only $0.13 \%$ of observations and the magnitude was small (8 doses or fewer); in these cases, we assumed the number of doses was zero.

We used line charts to visualize changes in medication dispensing over time by medication and hospital. We compared rates of medications use between unit type and hospital with use of relative difference and rate difference. Statistical analysis was performed with R (The R Foundation for Statistical Computing, 2018) using lubridate (2011), dplyr (2018), ggplot2 (2016), fmsb (2019), and forcats (2018).

\section{RESULTS}

A total of 1,542,225 inpatient days were analyzed, of which $60.4 \%$ were at hospital A. Internal medicine accounted for $23.5 \%$ of inpatient days, critical care for $11.7 \%$, and other units for $64.8 \%$.

\section{Overall Trends in Sleep Medication Use}

There were 351,131 dispensed doses of study medications (13\% melatonin, 43\% lorazepam, and 44\% zopiclone). Overall use of the three study medications per 1,000 inpatient days increased by $25.7 \%$ during the study.

Melatonin use increased by 71.3 doses per 1,000 inpatient days during 2013-2018, while zopiclone use decreased by 20.4 doses per 1,000 inpatient days (Table). Lorazepam use increased slightly by 3.5 doses per 1,000 inpatient days. All rate

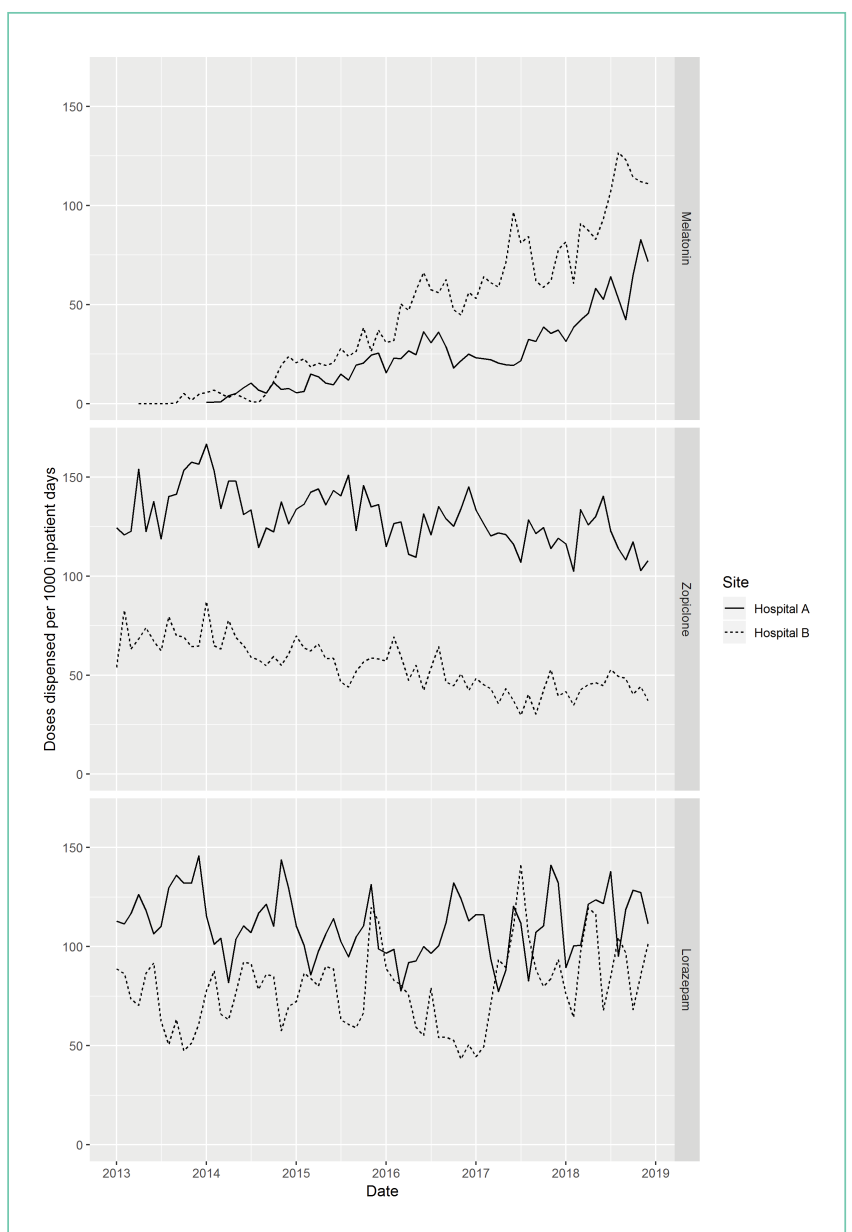

FIG. Monthly rate of medication dispensing by hospital site from January 2013 to December 2018. Each panel represents a different study medication.

differences reported in the results are statistically significant (Appendix Table).

\section{Unit Type Comparison}

Melatonin use was highest in critical care and internal medicine (50.9 and 48.4 doses per 1,000 inpatient days, respectively), compared with that in other units (19.3 doses per 1,000 inpatient days). Among critical care units, melatonin use was highest in medical-surgical units (67.4 doses per 1,000 inpatient days) and lower in cardiac and cardiovascular surgery units (24.6 and 18.3 doses per 1,000 inpatient days respectively). Zopiclone use was highest in critical care and other units (117.9 and 112.2 doses per 1,000 inpatient days, respectively) and lowest in internal medicine (57.0 doses per 1,000 inpatient days).

\section{Hospital Site Comparison}

Overall melatonin use was $65.4 \%$ higher at hospital B than at hospital A (42.4 vs 21.5 doses per 1,000 inpatient days; Figure). Zopiclone use was $81.7 \%$ lower at hospital B (54.6 vs 130.0 doses per 1,000 inpatient days).

When similar units were compared between hospitals, the trends were similar. For example, among internal medicine 
units, melatonin use was $66.7 \%$ higher at hospital B than at hospital A (64.4 vs 32.3 doses per 1,000 inpatient days).

\section{DISCUSSION}

During this 6-year study period of sleep medication use at two academic hospitals, overall use of melatonin, zopiclone, and lorazepam increased by $25.7 \%$. Melatonin increased from almost no use to more than 70 doses per 1,000 inpatient days. The increase in melatonin was not accompanied by a proportional decline in zopiclone, which only decreased by 20.4 doses per 1,000 inpatient days. Lorazepam use increased slightly. This suggests that melatonin is not simply being substituted for higher-risk sleep medications and is instead being given to patients who might not have received sleep medications otherwise.

There are a few potential explanations for the disproportionate increase in melatonin. Providers may be more liberal in prescribing melatonin for insomnia because of perceived greater safety, compared with other medications. Melatonin may also be prescribed for delirium, despite a lack of high-quality evidence. ${ }^{14}$ Interestingly, melatonin use has increased despite a paucity of evidence for its efficacy or safety in hospital. ${ }^{6}$ Considering the additional barriers that exist to ordering melatonin, a nonformulary medication at our institution, the magnitude of increase is even more striking.

Melatonin use was highest on internal medicine and critical care units. This may reflect patient differences (eg, older patients with more comorbid conditions might leave prescribers reluctant to use benzodiazepines), differences in the physical environment (eg, noise/lighting), differences in nursing practices (eg, intensity of monitoring or medication administration), or differences in prescribing.

Melatonin use was almost twice as high at hospital B as it was at hospital A. While the services differ at each hospital, the results were similar when comparing the same unit type (eg, internal medicine). Internal medicine units have similar (though not identical) patient populations and team structures at both hospitals, and residents rotate between hospitals. Attendings and nurses are based primarily at one hospital and their practice patterns might differ. Geriatricians have a stronger presence at hospital B. Higher zopiclone use at hospital A could explain lower melatonin use. Lastly, improvement initiatives may have contributed (eg, one unit at hospital B promoted melatonin in 2017).

\section{LIMITATIONS}

Our study has potential limitations. We studied dispensed rather than administered medications; however, numbers of doses dispensed but not administered are expected to be low because most unused doses are accounted for. By studying dispensing data, we might have underestimated the number of prescriptions (eg, if a patient was prescribed but refused a medication, this would not be captured). Our study did not examine medications after hospital discharge, although medications started in a hospital are often continued at discharge..$^{7,15}$
Our study could not determine indications for medication prescribing, and melatonin and lorazepam are both used for nonsleep indications. Our study could not differentiate between continuation of home medications and new prescriptions. Finally, the results may not be generalizable to other settings.

\section{CONCLUSION}

In this 6-year study of sleep medication use at two academic hospitals, we found that overall use of melatonin, zopiclone, and lorazepam increased by $25 \%$, predominantly because of markedly increased melatonin use. Given the current lack of high-quality evidence, further research on the use of melatonin in hospitalized patients is needed.

Disclosures: The authors declare that they have no conflicts of interest.

\section{References}

1. Wesselius HM, van den Ende ES, Alsma J, et al. Quality and quantity of sleep and factors associated with sleep disturbance in hospitalized patients. JAMA Intern Med. 2018;178(9):1201-1208. https://doi.org/10.1001/jamainternmed.2018.2669.

2. DePietro $\mathrm{RH}, \mathrm{Knutson} \mathrm{KL}$, Spampinato $\mathrm{L}$, et al. Association between inpatient sleep loss and hyperglycemia of hospitalization. Diabetes Care. 2017;40(2):188-193. https://doi.org/10.2337/dc16-1683

3. Inouye SK, Bogardus ST Jr, Charpentier PA, et al. A multicomponent intervention to prevent delirium in hospitalized older patients. N Engl J Med. 1999;340(9):669-676. https://doi.org/10.1056/NEJM199903043400901.

4. Ho A, Raja B, Waldhorn R, Baez V, Mohammed I. New onset of insomnia in hospitalized patients in general medical wards: incidence, causes, and resolution rate. J Community Hosp Intern Med Perspect. 2017;7(5):309-313. https://doi.org/10.1080/20009666.2017.1374108.

5. Rawal S, Kwan JL, Razak F, et al. Association of the trauma of hospitalization with 30-day readmission or emergency department visit. JAMA Intern Med. 2019;179(1):38-45. https://doi.org/10.1001/jamainternmed.2018.5100.

6. Kanji S, Mera A, Hutton B, et al. Pharmacological interventions to improve sleep in hospitalised adults: a systematic review. BMJ Open. 2016;6(7):e012108. https://doi.org/10.1136/bmjopen-2016-012108.

7. Gillis CM, Poyant JO, Degrado JR, Ye L, Anger KE, Owens RL. Inpatient pharmacological sleep aid utilization is common at a tertiary medical center. $J$ Hosp Med. 2014;9(10):652-657. https://doi.org/10.1002/jhm.2246.

8. Schroeck JL, Ford J, Conway EL, et al. Review of safety and efficacy of sleep medicines in older adults. Clin Ther. 2016;38(11):2340-2372. https://doi. org/10.1016/j.clinthera.2016.09.010.

9. Kolla BP, Lovely JK, Mansukhani MP, Morgenthaler TI. Zolpidem is independently associated with increased risk of inpatient falls. J Hosp Med. 2013;8(1):1-6. https://doi.org/10.1002/jhm.1985.

10. Buscemi N, Vandermeer B, Hooton N, et al. The efficacy and safety of exogenous melatonin for primary sleep disorders. a meta-analysis. J Gen Intern Med. 2005;20(12):1151-1158. doi:10.1111/j.1525-1497.2005.0243.x.

11. Clarke TC, Black LI, Stussman BJ, Barnes PM, Nahin RL. Trends in the use of complementary health approaches among adults: United States, 2002-2012. Natl Health Stat Report. 2015(79):1-16.

12. Erland LA, Saxena PK. Melatonin natural health products and supplements: presence of serotonin and significant variability of melatonin content. J Clin Sleep Med. 2017;13(2):275-281. https://doi.com/10.5664/jcsm.6462.

13. Brandt J, Alessi-Severini S, Singer A, Leong C. Novel measures of benzodiazepine and z-drug utilisation trends in a canadian provincial adult population (2001-2016). J Popul Ther Clin Pharmacol. 2019;26(1):e22-e38. https://doi. org/10.22374/1710-6222.26.1.3.

14. Siddiqi N, Harrison JK, Clegg A, et al. Interventions for preventing delirium in hospitalised non-ICU patients. Cochrane Database Syst Rev. 2016;3:CD005563. https://doi.org/10.1002/14651858.CD005563.pub3.

15. MacMillan TE, Kamali R, Cavalcanti RB. Missed opportunity to deprescribe: docusate for constipation in medical inpatients. Am J Med. 2016;129(9):1001. e1001-1007. https://doi.org/10.1016/j.amjmed.2016.04.008 